\title{
Epidermoid cyst in an intra-pancreatic accessory spleen
}

\begin{abstract}
The estimated prevalence of accessory spleen, most commonly found at the splenic hilum, is about $10 \%$ in the autopsy studies. The second commonly involved site is pancreas, and $1-2 \%$ of general population has intra-pancreatic accessory spleen (IPAS). Differential diagnosis of IPAS includes other solid pancreatic tumors, especially non-functioning pancreatic neuroendocrine tumors. Computed tomography scans and diffusion-weighted magnetic resonance imaging may accurately distinguish IPAS from other solid pancreatic lesions, with imaging features similar to the native spleen. Epidermoid cysts (EC) are common in the native spleen, but rarely found in IPAS. Differential diagnosis of the EC in IPAS includes other cystic lesions of the pancreas. Although the condition is extremely rare, an accurate pre-operative diagnosis can save a patient from major surgery given the benign nature of this condition. We present a case of an EC in an IPAS who underwent distal pancreatectomy with splenectomy for suspicion of malignancy.
\end{abstract}

Keywords: epidermoid cyst, intra-pancreatic accessory spleen, endoscopic ultrasound, hispanic woman
Volume 6 Issue 3 - 2017

\author{
Ali Raza,Anusha Thomas, Alberto O Barroso \\ Department of Medicine, Weill Cornell Medicine, USA
}

Correspondence: Ali Raza, Department of Medicine, Division of Gastroenterology, Houston Methodist Hospital, Weill Cornell Medicine, Houston campus, Houston, TX-77030, USA, Emailaraza@houstonmethodist.org

Received: December 19, 2016 | Published: February 27, 2017
Abbreviations: IPAS: Intra-Pancreatic Accessory Spleen; EC: Epidermoid Cysts; CT: Computed Tomography; MRI: Magnetic Resonance Imaging; EUS: Endoscopic Ultrasound; FNA: Fine Needle Aspiration

\section{Case}

A 32-year-old Hispanic woman presented with recent onset of moderate right upper quadrant abdominal pain. The pain was not associated with any aggravating or relieving factors and gradually resolved during her hospital stay. Past history was significant for obesity and hysterectomy. Physical examination showed normal vital signs and mild upper abdominal discomfort on deep palpation. Comprehensive laboratory work-up was unremarkable. A noncontrast computed tomography $(\mathrm{CT})$ of the abdomen showed a vague lesion in the pancreatic tail. A contrast-enhanced magnetic resonance imaging (MRI), done to better characterize the lesion, showed a 4-cm pancreatic tail mass, and a 1-cm cystic area in that mass (Figure 1). Endoscopic ultrasound (EUS) showed a 4-cm oval, hypo-echoic solid mass in the pancreatic tail, and a 1-cm an-echoic (cystic) area within that mass. Fine needle aspiration of the mass showed wellcircumscribed lymphoid aggregates, without monoclonal expansion, mixed with red blood cells. For concerns of malignancy, the patient underwent distal pancreatectomy with splenectomy. The resected specimen showed a well-circumscribed 4-cm red-brown lobulated mass representing ectopic spleen with reactive lymphoid hyperplasia (Figure 2) (Figure 3), and a 0.7-cm multilocular benign squamous cyst within the accessory spleen (Figure 4). Post-operative course was unremarkable and the patient was discharged home in a stable condition.

\section{Discussion}

The prevalence of accessory spleen, most commonly found at the splenic hilum, is about $10 \%$ in the autopsy studies. ${ }^{1}$ Intra-pancreatic accessory spleen (IPAS) originates from splenic remnants in dorsal pancreatic duct during embryogenesis, and occurs in $1-2 \%$ of the general population. ${ }^{2}$ Epidermoid cysts are infrequent primary (true) splenic cysts and usually do not occur in the accessory spleen. First case of an epidermoid cyst within an IPAS was described in $1980,{ }^{3}$ with only 36 cases described so far. ${ }^{4,5}$ Epidermoid cysts in IPAS occur with equal incidence among men and women, predominantly in the middle age group, and always located in the pancreatic tail. ${ }^{4}$ Clinically, most patients remain asymptomatic and are diagnosed incidentally with imaging studies. However, there are a handful of patients who may present with nausea, vomiting or abdominal pain.

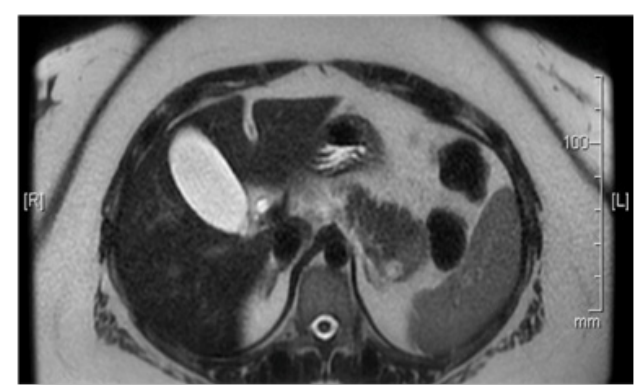

Figure I The T2 diffusion-weighed cross sectional images of the MRI. There is $4 \mathrm{~cm}$ hypo-intense pancreatic tail lesion, and a $\mathrm{Icm}$ T2 bright cystic structure within that lesion.

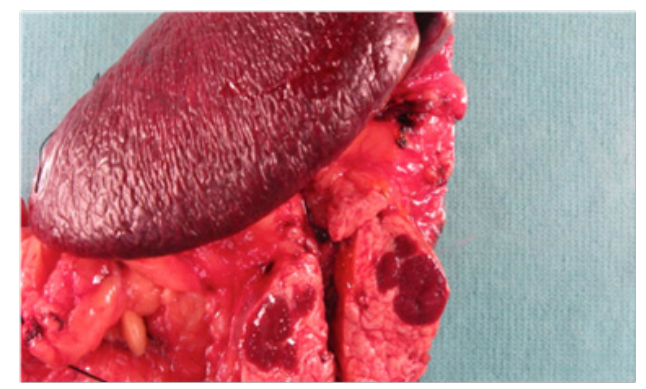

Figure 2 Surgical specimen showing a $4 \mathrm{~cm}$ structure with in the pancreatic tail (IPAS) that is similar in to the native spleen. 


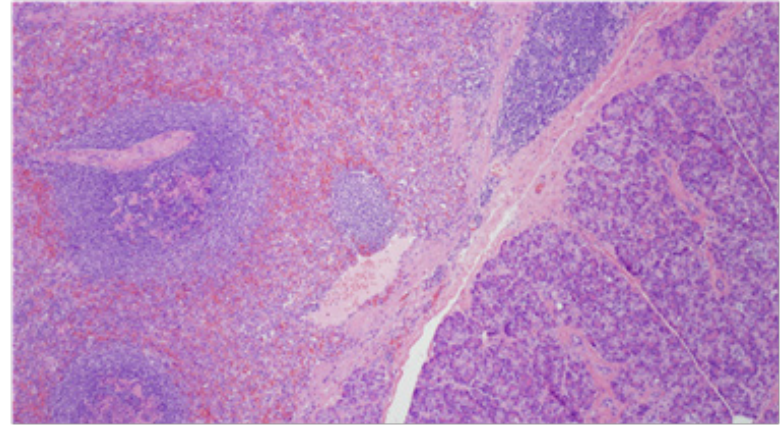

Figure 3 Low power cross section through the resected specimen shows a benign pancreatic tissue on the right in purple, separated from the lymphoid aggregates or white pulp and red pulp of the accessory spleen on the left.

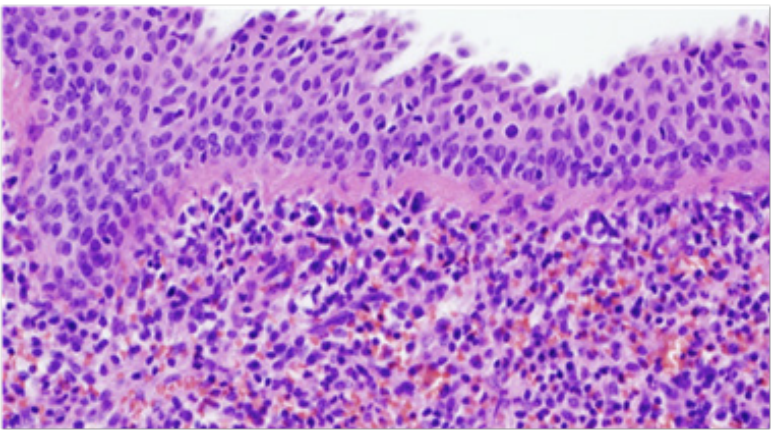

Figure 4 High power H\&E stain of the small I cm cystic lesion within accessory spleen shows a stratified squamous epithelium lining the cyst.

Despite their rarity, epidermoid cysts in IPAS should be recognized as a differential diagnosis of pancreatic tail lesions in appropriate clinical setting. Non-invasive imaging modalities (CT or MRI) may aid in diagnosing the epidermoid cyst in the IPAS. On multi-phasic dynamic CT scan, IPAS has the same attenuation as native spleen. Since attenuation of spleen is higher than pancreas, IPAS appears brighter than pancreas on all three phases of CT scan, this is in contrast to other hypervascular lesions of pancreas that may become iso- or hypo-attenuating to the adjoining pancreas on the venous phase. On MRI, epithelial cysts commonly show low signal intensity on T1weighted images and high signal on T2-weighted images. The ectopic splenic tissue shows a spleen-like signal on diffusion-weighted images. Adequate amount of splenic tissue around the cyst makes the diagnosis of epidermoid cyst in IPAS easy, otherwise diagnosis before surgery remains challenging. ${ }^{6}$

Endoscopic ultrasound (EUS) guided fine needle aspiration (FNA) is very important in the initial diagnosis of IPAS, and thus may save an unnecessary surgery. Typical FNA findings show heterogeneous population of lymphocytes often with a background of eosinophils, histiocytes and plasma cells, as well as small blood vessels. Perhaps the most striking feature of EUS-FNA is positive staining of the cellblock for $\mathrm{CD} 8$, which is a marker for splenic endothelial cell lining. This staining is thought to be a confirmatory test for IPAS, since systemic endothelial cells and hemangiomas do not stain positive with CD8. ${ }^{7}$

\section{Acknowledgments}

None.

\section{Author contributions}

Ali Raza: Reviewing and writing the manuscript

Anusha Thomas: Manuscript writing and data gathering

Alberto O Barroso: Critically reviewed the manuscript

\section{Informed consent}

Informed consent was obtained from the patient for publication of images and related information.

\section{Conflicts of interest}

The authors declare there is no conflict of interests.

\section{Funding}

None.

\section{References}

1. Mortelé KJ, Mortelé B, Silverman SG. CT features of the accessory spleen. AJR. 2004;183:1653-1657.

2. Halpert B, Alden ZA. Accessory spleen in or at the tail of the pancreas. A survey of 2,700 additional necropsies. Arch Pathol. 1964;77:652-654.

3. Davidson ED, Campbell WG, Hersh T. Epidermoid splenic cyst occurring in an intrapancreatic accessory spleen. Dig Dis Sci. 1980;25(12):964-967

4. Zavras N, Machairas N, Foukas P, et al. Epidermoid cyst of an intrapancreatic accessory spleen: a case report and literature review. World J Surg Oncol. 2014;12:92.

5. Saunders TA, Miller TR, Khanafshar E. Intrapancreatic accessory spleen: utilization of fine needle aspiration for diagnosis of a potential mimic of a pancreatic neoplasm. J Gastrointest Oncol. 2016;7(Suppl 1):S62-S65

6. Van Dijck WPM, Groot VP, Brosens LAA, et al. Rare case of an epithelial cyst in an intrapancreatic accessory spleen treated by robotassisted spleen preserving distal pancreatectomy. Case Reports in Gastrointestinal Medicine. 2016:9475897.

7. Rodriguez E, Netto G, Li QK. Intrapancreatic accessory spleen: a case report and review of literature. Diagn Cytopathol. 2013;41:466-469. 